\title{
Modelling and identification of linear discrete systems using least squares method
}

\author{
Peter Šarafín*, Martin Húdik*, Martin Revák*, Samuel Žák* and Peter Ševčík* \\ *Faculty of Management Science and Informatics, \\ Univerzitná 8215/1, Žilina 01026 \\ Email: see http://www.fri.uniza.sk
}

\begin{abstract}
In control applications, we often encounter systems that respond to the change of control signal in an undesirable way. To adjust system output, there arises the need to know system parameters, so the identification has to be performed. The aim of this paper is to compare upstanding identification error that is the consequence of dataset size, input signal type, and quantization error occurring in the signal. The experimental part of this paper presents the results measured on the real device and shows the identification results.
\end{abstract}

\section{INTRODUCTION}

$\mathbf{T}$ HE purpose of identification is to experimentally determine the structure and complexity of the model. After determining the structure and complexity of the model, an appropriate method is used to estimate the unknown system model parameters.

The first step in system identification from experimental data is modelling. The behaviour of the model is determined by the structure of the system and by the properties of the equations describing the relations of the action members. The way the individual subsystems are interconnected and how they operate is described by the overall system and its behaviour. The behaviour of the system obtained using equations describing the physical model can be described in detail by a set of algebraic and differential equations.

\section{SYSTEM IDENTIFICATION BACKGROUND}

In automated control and signal processing, we understand the dynamic system model as a mathematical description of the relationship between inputs and outputs of the system. Based on this context, it is possible to determine the system transfer function and thus to identify the system. Basic methods of identification may include methods such as transition and impulse characteristics. The excited input has the character of a single jump or a unit pulse, and the output signal states the model. The application of these techniques is simple, not very susceptible to noise. Another drawback of using these techniques to identify the system is the need to introduce a unit jump / input impulse, which is undesirable for some systems [1]. For this reason, we are also addressing other systems identification approaches that are described in the following text.

The gradient method and the least squares method can be used to estimate the parameters of any linear system [2], [3],
[5]. For simplicity and clarity, consider the transport delay $d=1$. Equation (1) states that

$$
y(k)=\varphi^{T}(k-1) \theta,
$$

where

$$
\begin{aligned}
& \theta=\left[\begin{array}{ll}
-a_{1} \ldots-a_{n} & b_{0} \ldots b_{m}
\end{array}\right]^{T}, \\
& \varphi(k-1)=[y(k-1) \ldots y(k-n) \quad u(k-1) \ldots \\
& \ldots u(k-m)]^{T} \text {. }
\end{aligned}
$$

$\theta$ is the vector of the system parameters we are trying to determine and $\varphi(k-1)$ is called a regression vector as it is made up of previous system inputs and outputs that affect the current system output value. When determining the correct system parameter values, it is necessary to determine the initial estimate $\hat{\theta}(0)$. Then the parameter values are so adjusted that the difference between the estimated system output $\hat{y}(k)=\varphi(k-1)^{T} \hat{\theta}(k-1)$ and the actual output of the system $y(k)=\varphi(k-1)^{T} \theta$ is minimized in time. The task of adaptation is thus minimization of the error between the difference of the expected and the actual output (3).

$$
e(k)=|y(k)-\hat{y}(k)|=\left|\varphi(k-1)^{T} \theta-\varphi(k-1)^{T} \hat{\theta}(k-1)\right|
$$

The adaptation scheme is illustrated in Fig. 1.

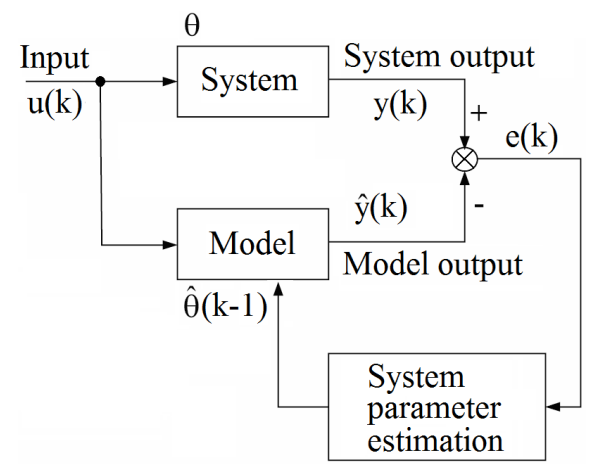

Fig. 1. The adaptation scheme of model parameters.

\section{Modelling AND SIMUlation OF THE ACCELEROMETER BASED SYSTEM IDENTIFICATION}

In order to observe the behaviour of the model, the model must first be conducted. At first, we need to define the properties of the system that we are modelling. When modelling 
a weakly damped linear discrete system, it is necessary to determine the system's own frequency and the damping ratio. A relationship

$$
\frac{d^{2} x(t)}{d t^{2}}+2 \zeta \omega_{n} \frac{d x(t)}{d t}+\omega_{n}^{2} x(t)=f(t)
$$

evokes that the knowledge of these parameters is sufficient to describe the second order differential equation and hence the ideal stabilized system [7]. To solve this differential equation, Laplace transform means were preferred for clarity, by means of which it is possible to shift from a mathematical model in the form of a differential equation to a system description by means of $F(s)$

$$
F(s)=\frac{\omega_{n}^{2}}{s^{2}+2 \zeta \omega_{n} s+\omega_{n}^{2}} .
$$

After transforming into a z-plane we get a defined discrete model describing the system we want to simulate. The advantages of such writing include a relatively simple determination of the location of the zeros and poles based on the polynomial numerator and denominator of the system.

In order to verify the suitability of the identification method, it is adequately to generate several kinds of input signals to excite the modelling system. Selected control signals include unit pulse, unit jump, harmonic signal, or a combination thereof. Due to the fact that we model the data obtained from the accelerometer, it is necessary to set certain limitations. The simulated control signals are suitable for a certain width. We decided to represent the result by a 12-bit binary number. These signals were also affected by quantization noise. Quantum error has the character of white noise and normal distribution [7]. Based on the above, noise is generated with a normal distribution in the range determined by the sensitivity of the measurement and the range and is then added to the input and output signals.

\section{SYSTEM IDENTIFICATION USING THE LEAST SQUARES METHOD}

To use this method for identification, it is necessary to have at least $P$ data from the data set (6), whereby

$$
P=n+m,
$$

where $n$ is the order of denominator and $m$ is the order of numerator [3], [5]. Thus, the second order transfer function is in shape

$$
\hat{y}(z)=\frac{b_{0}+b_{1} z^{-1}}{1+a_{1} z^{-1}+a_{2} z^{-2}} u(z)+e(z),
$$

where the measurable magnitudes are only the input $U(z)$ and the output of the model $Y(z)$. Systems with higher order can be represented analogically. The aim is to identify coefficients of the numerator $b$, the denominator $a$ of the system, but about the random error $e(n)$, we know only that it has a Gaussian distribution, the character of the white noise, and a zero mean value [4]. The equation (7) can be rewritten into the analytical form

$$
\begin{array}{r}
{\left[\begin{array}{cccc}
u(k) & u(k-1) & -\hat{y}(k-1) & -\hat{y}(k-2) \\
u(k+1) & u(k) & -\hat{y}(k) & -\hat{y}(k-1) \\
u(k+2) & u(k+1) & -\hat{y}(k+1) & -\hat{y}(k) \\
u(k+3) & u(k+2) & -\hat{y}(k+2) & -\hat{y}(k+1)
\end{array}\right]\left[\begin{array}{l}
b_{0} \\
b_{1} \\
a_{1} \\
a_{2}
\end{array}\right]+} \\
+\left[\begin{array}{c}
e(k) \\
e(k+1) \\
e(k+2) \\
e(k+3)
\end{array}\right]=\left[\begin{array}{c}
\hat{y}(k) \\
\hat{y}(k+1) \\
\hat{y}(k+2) \\
\hat{y}(k+3)
\end{array}\right] .
\end{array}
$$

By stating

$$
\begin{gathered}
A_{p}=\left[\begin{array}{cccc}
u(k) & u(k-1) & -\hat{y}(k-1) & -\hat{y}(k-2) \\
u(k+1) & u(k) & -\hat{y}(k) & -\hat{y}(k-1) \\
u(k+2) & u(k+1) & -\hat{y}(k+1) & -\hat{y}(k) \\
u(k+3) & u(k+2) & -\hat{y}(k+2) & -\hat{y}(k+1)
\end{array}\right], \\
\theta_{p}=\left[\begin{array}{l}
b_{0} \\
b_{1} \\
a_{1} \\
a_{2}
\end{array}\right], e_{p}=\left[\begin{array}{c}
e(k) \\
e(k+1) \\
e(k+2) \\
e(k+3)
\end{array}\right] \text { and } \hat{y}_{p}=\left[\begin{array}{c}
\hat{y}(k) \\
\hat{y}(k+1) \\
\hat{y}(k+2) \\
\hat{y}(k+3)
\end{array}\right]
\end{gathered}
$$

we obtain

$$
\hat{y}_{p}=A_{p} \theta_{p}+e_{p} .
$$

The minimum number of required data is usually small. For the second order system, at least four consecutive data from the data set must be available. The complexity of the system also increases the minimum number of data points needed for sufficient accuracy. This has the effect that $P$ is much larger (the number of rows of the $A$ matrix grows). A larger $P$ should provide more accurate definition of system parameters. The equation used to find optimal parameters (the smallest error) is

$$
\hat{\theta}_{p}=A_{p}^{+} \hat{y}_{p}
$$

where $A_{p}^{+}$represents a pseudo-inverse matrix to $A_{p}$ (12) [6].

$$
A_{p}^{+}=\left(A_{p}^{T} A_{p}\right)^{-1} A_{p}^{T}
$$

From the definition of this method, it can be deduced that choosing a larger number of input-output sample pairs leads to a more accurate system determination (Fig. 2).

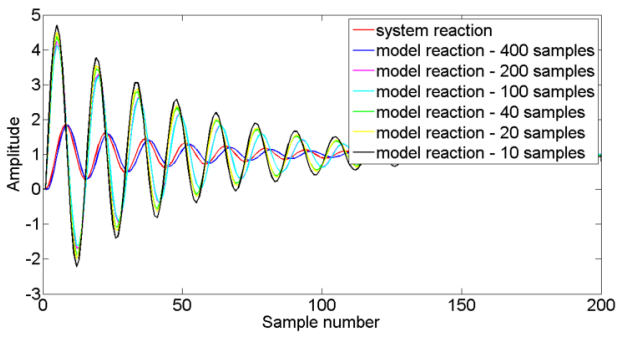

Fig. 2. Comparison of the system and models obtained using different number of samples. 
The error we commit when identifying depends on the number of samples used to determine the model parameters (Tab. I). However, it is important to be aware of the computational difficulty that grows with the addition of additional samples to be classified.

TABLE I

A COMPARISON OF OVERALL ERROR OF MODELS USING DIFFERENT NUMBER OF SAMPLES.

\begin{tabular}{|c|c|}
\hline Number of used samples & RMS error \\
\hline 10 & 295,4 \\
\hline 20 & 202,4 \\
\hline 40 & 196 \\
\hline 100 & 172,3 \\
\hline 200 & 190,9 \\
\hline 400 & 3,905 \\
\hline
\end{tabular}

When designing the simulation, we considered the appropriate choice of parameters. Based on the conducted initial experiment, we considered sampling frequency $400 \mathrm{~Hz}$, damping ratio 0.05 and a resonant frequency of $28 \mathrm{~Hz}$ as the parameters of second order system.

The nature of the input signal has also an impact on the system identification. In some cases, the process of determining system parameters can not be defined as the reaction of the system to a signal in the prescribed form, which results in a limitation resulting from the suitability of the control signal being used. The response of the system to various input signals (Fig. 3) determines the parameters of the identified system differently. In our case, we chose the size of the identifying set to 20 input and 20 output data. The error that occurs in the input and output signals reaches a maximum of $1 \%$ of the range.

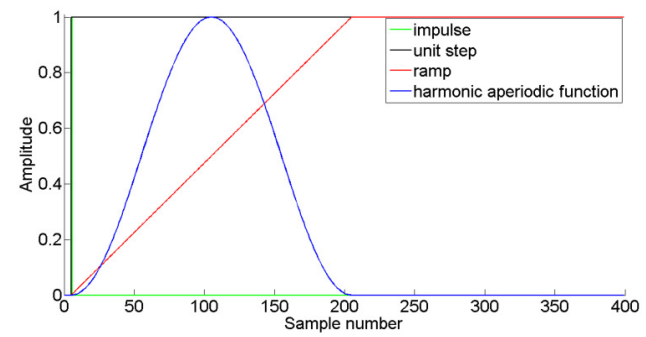

Fig. 3. Behaviour of selected control signals.

Adequacy of the use of different types of control signals for identification purposes may be expressed as a root mean square error (RMS error) (Table II). From the test results, it can be observed that in the least squares method it is advisable to use jump control signals for identification. Introducing an error in the input and output signal representing noise from the sensor device greatly affects the quality of the identification.

The simulation showed, that if no error occurrs in the signal, this method has excellent results. By adding noise to the ideal signal, the result becomes less accurate, which in some cases has led to a poor classification of the system. White noise with normal distribution is generated and has a zero average value within a certain range and is then added to the input
TABLE II

A COMPARISON OF OVERALL ERROR OF MODELS USING DIFFERENT CONTROL SIGNALS.

\begin{tabular}{|c|c|}
\hline Identification control signal & RMS error \\
\hline unit pulse & 1,161 \\
\hline unit step & 0,9191 \\
\hline ramp & 1,344 \\
\hline harmonic aperiodic function & 2,081 \\
\hline
\end{tabular}

and output signal (Figure 4). Thus affected input and output signal are used in the identification process.

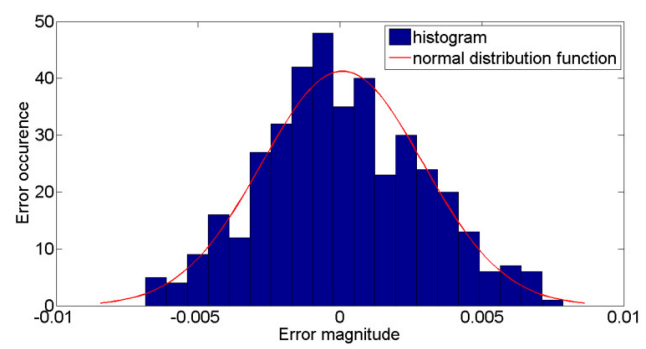

Fig. 4. Histogram and normal distribution function of generated error.

By enhancing the set of input and output signal samples, the quality of identification improves, but not sufficiently. The influence of selected noise levels at the value of the twenty inout samples used for identification is shown in Tab. III.

TABLE III

THE IMPACT OF NOISE ON THE QUALITY OF IDENTIFICATION.

\begin{tabular}{|c|c|}
\hline established error & RMS error \\
\hline $0 \%$ & 0,009812 \\
\hline $1 \%$ & 2,266 \\
\hline $2 \%$ & 2,401 \\
\hline $3 \%$ & 2,484 \\
\hline $4 \%$ & 2,547 \\
\hline $5 \%$ & 2,606 \\
\hline
\end{tabular}

Practical application has shown that when eliminating residual vibrations, it is important to determine the exact frequency of the system as accurately as possible. These results served as a basis for modifying the identification of systems in the frequency domain.

\section{EXPERIMENTAL RESULTS}

Based on the need to control the coin hopper tester device, we have chosen this device as a system that needed to be identified. When identifying the dynamic properties of the system, we used printed circuit board featuring an accelerometer (Fig. 5).

As a control element, ATmega168 microcontroller was used. Its task was to provide the LSM303DLHC accelerometer configuration and send the recorded data via the RF module RFM73 to eliminate the undesirable phenomena associated with the use of wire communication. The accelerometer has been set to measure acceleration in the $x, y$ and $z$ axes. The sampling rate was set to $400 \mathrm{~Hz}$, which corresponds to the 


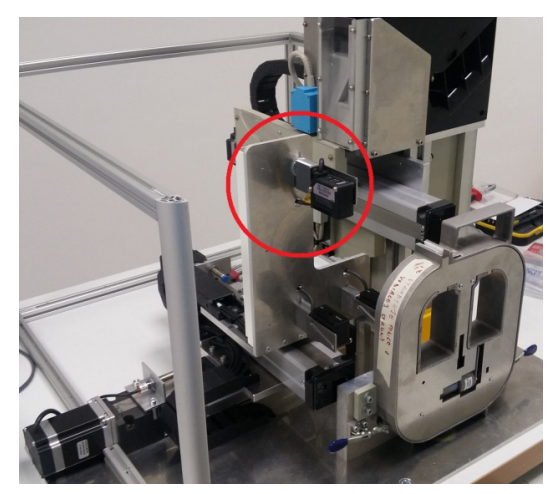

Fig. 5. Coin hopper tester device with marked critical part.

maximum possible recording speed when the accelerometer is used.

As the receiver, we used a PCB with ATmega8A microcontroller. The role of this microcontroller was to receive the recorded data sent to the RFID measurement module via the RFM73 RF module and then to send results using the UART peripheral. In order to be able to continue working with this data on the computer, we used a communication interface converter to convert between USB and UART peripherals.

The measuring device was positioned such that the movement of the arm manifested mainly in one axis of the accelerometer (Fig. 6). On the PC, the incoming data has been aggregated into a file so it could be analyzed. We used Matlab to analyze measured signals representing acceleration in individual axes of the accelerometer.

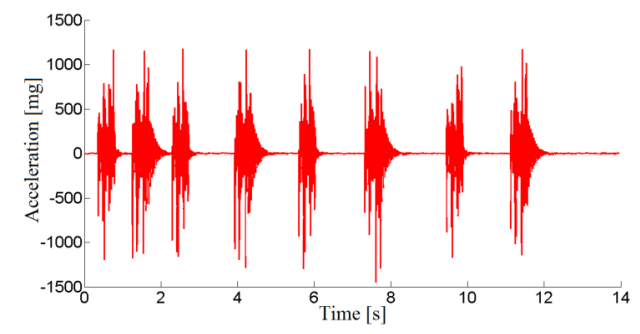

Fig. 6. Data obtained from critical accelerometer axis.

Using the least squares method, we determined the zeroes and poles of the system. After a series of parameter estimations, when it was not possible to minimize the error below the set level (Fig. 7), a situation occurred, in which the analyzed signal represented residual vibrations (Fig. 8). When approximating such a signal, we made errors as shown in Fig. 9.

\section{CONCLUSION}

This paper presents error comparison when identification using least squares method is applied. The obtained results confirm that the error we commit by identification directly depends on the number of samples used for model parameters determination.

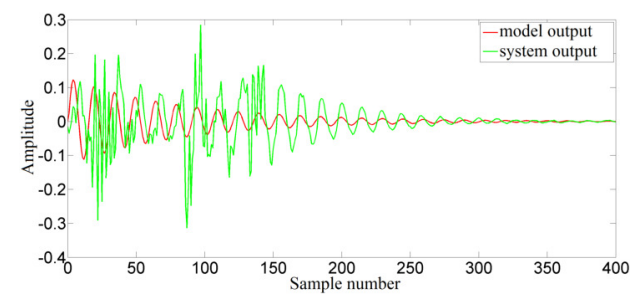

Fig. 7. Comparison of system and model reaction.

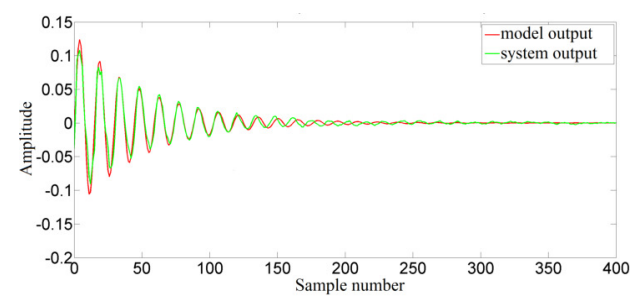

Fig. 8. Comparison of system and model reaction.

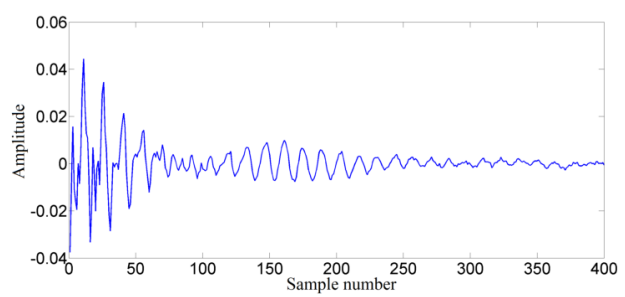

Fig. 9. Satisfactory identification of system parameters - identification error.

Another aspect that needs to be noticed is the control signal that is used in identification process. The deviation of model parameters results in the error on system output. It was shown that the input signal should generate adequate excitation of the system, otherwise the error significantly affects the parameter estimation.

\section{REFERENCES}

[1] Karpiš O., Miček J., Olešnaníková V.: Using of compressed sensing in energy sensitive WSN applications FedCSIS 2015, September 13-16, Lodz, Poland. - ISBN 978-83-60810-65-1. - pp. 1233-1238, http://dx.doi.org/10.15439/2015F167.

[2] Skovranek T., Despotovic V.: Identification of Systems of Arbitrary Real Order: A New Method Based on Systems of Fractional Order Differential Equations and Orthogonal Distance Fitting, ASME 2009 Inter. Design En. Tech. Conf. and Computers and Information in Engineering Conference, pp. 1063-1068, 2009.

[3] McKelvey T.: Least Squares and Instrumental Variable Methods, Control Systems, Robotics, and Automation, in EOLSS, Developed under the auspices of the UNESCO 2004.

[4] Guo W.: Modelling and Simulation of a Capacitive Micro-Accelerometer System, Proceedings of the 33rd Chinese Control Conference July, Nanjing, China, 2014

[5] Švarc I., Matoušek R., Šeda M., Vítečková M.: Automatické řízení, Brno: CERM, ISBN: 978-80-214-4398-3, 2011.

[6] Golan J.: Moore-Penrose pseudoinverses, The linear algebra a beginning graduate student ought to know, Springer Netherlands, pp. 441-452, 2012.

[7] Miček J., Jurečka M.: Moderné prostriedky implementácie metód číslicového spracovania signálov 1, Žilina: EDIS, ISBN: 978-80-554-07142, 2013. 